\title{
Influence of prenatal alcohol and smoke exposure on neonatal vagal tone in response to head-up tilt
}

\author{
Nicolò Pini, Maristella Lucchini, William P. Fifer, Michael M. Myers, and Maria G. Signorini
}

\begin{abstract}
This paper investigates differences in the parasympathetically mediated heart rate response to head-up tilt in two populations of newborns. One group was unexposed to any drug during pregnancy, the other was exposed to both alcohol and smoking in utero. Four different estimates of vagal tone were calculated. These indexes quantify vagal tone magnitude in four different domains: time, frequency, complexity and phase. Control group (CG) results across all parameters show a consistent physiological response to an orthostatic tilt consistent with vagal withdrawal. On the other hand, infants in the exposed group (EG) did not express a decrease in vagal measures following tilt.
\end{abstract}

\section{INTRODUCTION}

Sudden infant death syndrome (SIDS) is defined as the unexpected death of a seemingly healthy infant under 12 months of age, which remains unexplained after complete autopsy and investigation [1]. It is one of the leading causes of death during the first year of life in developed countries, but the exact etiology of SIDS remains unknown. The most influential model is called Triple Risk Hypothesis and states that the probability of SIDS is increased when the infant: 1) has an underlying vulnerability such as prematurity or in utero exposure to tobacco smoke, alcohol, and/or other drugs 2 ) is in an unstable developmental period (2-4 months associated with the highest risk), and 3) is exposed to exogenous stressors, such as prone sleeping position, bed sharing [2]. These risk factors affect the infant's ability to face internal and external stressors and may also contribute to autonomic nervous system (ANS) failure to maintain homeostatic control following physiologically challenging events [1].

In this study attention was focused on the effects of prenatal alcohol and smoke exposure. Associations between exposure and increased two- to five-fold risk of SIDS has been reported [3]. It has been reported that heavy prenatal alcohol exposure, increases the risk of SIDS by sevenfold. The underlying hypothesis describes abnormalities in the control of homeostatic functions by the developing brainstem [4]. Exposure to nicotine and alkaloid in cigarettes may contribute to Harper et al.'s proposed model, which states that SIDS infants are less able to arouse and respond to potential life-threatening physiological challenges [5].

Nicolò Pini, Maristella Lucchini, and Maria G. Signorini are with Dipartimento di Elettronica, Informazione e Bioingegneria (DEIB), Politecnico di Milano, Milano, MI 20133 Italy (corresponding author email: nicolo.pini@polimi.it).

William P. Fifer, and Michael M. Myers are with the Departments of Psychiatry and Pediatrics, Columbia University Medical Center, New York, NY 10032 USA.
Graded head-up tilt has been widely used as a test to observe and quantify autonomic control. The tilt should elicit a shift of the sympathovagal balance toward a sympathetic activation and parasympathetic withdrawal [6]. Several indexes have been proposed to quantify sympathetic and parasympathetic activity. However, there is no current consensus for standardized measures of vagal activation/withdrawal in infants [7]. In addition, newborns spend most of their time asleep, either in active or quiet sleep state while on the other hand, tilt is performed in wake state in adults.

Time domain quantification of vagal response to head up tilt in unexposed infants has been reported by Myers at al. [8]. A measure of beat-to-beat variability (RMSSD) showed significant decreases following head-up tilts, regardless of sleep state. Another method employed to quantify vagal activity is the computation of High Frequency (HF) spectral component of Heart Rate Variability (HRV) Power Spectral Density (PSD) [6], [9]. An indirect but quantifiable effect of respiration on beat-to-beat vagal control on $\mathrm{HR}$ is termed Respiratory Sinus Arrhythmia (RSA) [10]. RSA is determined by both tonic and phasic processes, with the tonic branch more related to vagal control. Medullary respiration control centers and pulmonary stretch receptors also contribute to RSA. Thus, neural and mechanical contributions are physiologically coupled and may not be easily dissociated. Furthermore, the relative contributions to RSA may vary across behavioral states, with a prevalence of ANS control during rest/sleep state [10].

This investigation entails a novel application of parameters of autonomic function control to characterize the immature neonatal ANS, whose cardiorespiratory interactions are not yet stable. Multiple measures of vagal withdrawal were investigated: traditional time/frequency domain estimates along with novel complexity measures. The analysis was performed on two cohort of infants, unexposed newborns and those exposed in utero to both alcohol and smoking. The combined use of these indexes suggested different regulatory dynamics in the two groups and opens to novel perspective in physiological and clinical SIDS risk assessment.

\section{MATERIALS AND METHODS}

\section{A. Subjects and data collection}

The subjects analyzed were enrolled in the Safe Passage study, the Ethical Review Boards approved all experimental procedures involving human subjects as described in [11]. They were recruited at Tygerberg Hospital and affiliated clinics in Bellville, Cape Town, South Africa. Infants were routinely discharged from the hospital less than 24 hours 
after delivery, they returned within 48-96 hours for assessment. At this visit, nurses recorded electrocardiogram (ECG), respiration, blood pressure (BP), and cortical brain activity (EEG) during sleep. Data recordings were approximately 1-hour long and occurred approximately 30 minutes after eating. The standardized procedure consisted of a 10-minutes baseline period and three rapid ( $\sim 3-5$ seconds) $45^{\circ}$ head-up tilts, while the infant was in the prone position [2] as it may accentuate the response evoked by tilting. Each tilt session is organized in: 30 -sec just prior tilt (B); 15 seconds block after the infant reached head-up position (I); three per 30-seconds (T1, T2, T3) before returning to flat position and repeated three time during 1-hour recording. The study analyzes data in first tilt session out of the three performed and the I block was not considered due to its lack of stationarity.

ECG was recorded at $500 \mathrm{~Hz}$ and respiratory tracings were simultaneously collected using a respiratory inductance belt, which was placed around each infant's chest and acquired at of $20 \mathrm{~Hz}$. A modification of the Timeline Follow-Back Interview (TLFB) was employed by nurses to guide mothers' self-reported estimation of their tobacco and alcohol consumption during pregnancy [11]. Based on the assessment, subjects were divided in: i) CG: no prenatal exposure to alcohol or tobacco smoke; ii) EG: prenatal exposure to both substances during the first, second or third trimester of pregnancy. Mothers in EG had on average more than 25 drinks and smoked consistently during the whole duration of their pregnancy. Inclusion criteria for the cohorts in this study were: full-term infants (gestational age (GA) at birth $\geq 37$ weeks), birthweight $\geq 2500 \mathrm{~g}$, South African mixed ancestry, no resuscitation at birth or admission to the Neonatal Intensive Care Unit (NICU), no prenatal exposures to drugs of abuse, no evidence of diabetes, hypertension, or pre-eclampsia during pregnancy in maternal medical records.

The total number of infants in the CG is $15(3 \mathrm{M}-12 \mathrm{~F})$, Gestational Age (GA) is $39.7 \pm 1.07$ weeks while in the EG the total number is $13(6 \mathrm{M}-7 \mathrm{~F})$, GA is $39.2 \pm 0.83$ weeks. Infants sleep states were classified into Active Sleep (AS) and Quiet Sleep (QS), Awake (A) or Indeterminate (I) by a Matlab automate algorithm [12]. In this study, the reported analysis was performed exclusively periods classified as QS.

\section{B. Signal processing}

The ECG signal was filtered to remove electrical line noise using a $50 \mathrm{~Hz}$ notch filter. The RR peaks were detected on the ECG with the Pan-Tompkins algorithm. An adaptive filter was then applied to remove ectopic beats or artifacts in the extracted RR series. RR intervals with a duration $<0.300 \mathrm{~s}(200 \mathrm{bpm})$ or $>0.667 \mathrm{~s}(90 \mathrm{bpm})$ as well as adjacent intervals with a relative increase $>15 \%$ were discarded from further analyses.

The respiration signal was band-pass filtered in the range 0.05-2 Hz. The Inter Breath Intervals (IBI) series, extracted based on respiration onsets, was corrected with an approach similar to RR series. Breath-to-breath intervals $<0.5$ seconds (120 breaths/minute) or $>2.0$ seconds ( 30 breaths/minute) were excluded.

\section{Extracted parameters}

Several quantitative estimators of vagal activation and/or withdrawal are reported in the literature. Given the lack of consensus for which measures are best, four different estimators along with their performances have been investigated in this study.

- RMSSD [s] (time domain) : the square root of the mean of the sum of the squares of differences of RR series, a time-domain measure of short-term components of HRV [9].

- HF_power $\left[\mathbf{s}^{2}\right]$ (frequency domain): the RR series was resampled at $5 \mathrm{~Hz}$ and its Power Spectral Density (PSD) was obtained by means of Welch PSD estimation. The spectrum was computed using 20-seconds overlapping Hamming windows. The HF frequency of HRV spectrum was defined as the frequency associated to the power in the window [mean breathing frequency $-0.1 \mathrm{~Hz}$; mean breathing frequency $+0.1 \mathrm{~Hz}]$ where mean breathing frequency is the inverse of mean IBI [9].

- Quadratic Sample Entropy (QSE) [bits] (complexity domain): this measure overcomes the limitations related to the univocal definition of the tolerance parameter $r$ when computing Sample Entropy (SampEn) [13]. QSE is defined in (1) as:

$$
Q S E=\operatorname{SampEn}(m, r)+\log (2 r)
$$

$r$ is optimally varied until a priori defined number of matches are observed given the embedding dimension $\mathrm{m}$. In this study, $m$ is set to 1 and initial value of $r$ is set to 0.1 *standard deviation of analyzed RR series.

- RSA_amplitude [s] (phase domain): is computed based on the method reported by Bartsch in [14]. In this work, the phase of the respiratory signal $\phi_{r}$ is computed using the DAMOCO toolbox [15]. The protophase of respiration signal is obtain using Hilbert transform and further corrected to obtain $\phi_{r}$. The series of relative heartbeat distances, obtained considering windows of two consecutive breaths $\phi_{r} \in[0,4 \pi]$, is fitted with a leastsquare sinusoidal approach. The RSA_amplitude is defined as the amplitude of the derived sinusoid.

The extraction of the four parameters provide a set of variables for each subject in each of the four tilt blocks, e.g. RMSSD_B, RMSSD_T1, RMSSD_T2, and RMSSD_T3.

\section{Statistical analysis}

The distribution of each parameter was tested for normality by means of Shapiro-Wilk test and values of skewness and kurtosis were computed. The variables RMSSD, HF_power and RSA_amplitude failed the above described normality tests thus, they were log transformed (ln_RMSSD, ln_HF_power, and ln_RSA).

The statistical analysis was performed using repeated measures ANOVA. This test aims at evaluating the withinsubject differences comparing the four time points corresponding to tilt blocks in CG and EG separately. As a consideration for further analysis, the overall extracted parameter distribution (regardless tilt blocks) can be considered approximately normally distribute, thus the 
requirement of normality for repeated measures ANOVA stands for both CG and EG.

\section{RESULTS}

The mean \pm standard deviation of parameters for each tilt block, F and p-value of Greenhouse-Geisser tests are reported in Table I. Each parameter was analyzed having tilt block as within-subjects factor. Statistical analysis shows a significant within-subjects effect of tilt block on all extracted parameters in the CG. Left panel of Figure 1 show the mean trend of the extracted parameters for $\mathrm{CG}$. The computed parameters show a consistent decreasing in vagal magnitude in the comparison of BvsT1 and BvsT2. On the other hand, the vagal activation pattern in segment $\mathrm{T} 2$ and $\mathrm{T} 3$ exhibit a flat trend.

To investigate the statistical significance of the reported differences between pairs of tilt blocks, a series of post-hoc tests were performed. The pairwise comparisons did not show any difference between BvsT1, BvsT3, T1vsT2, T1vsT3 or T2vsT3. However, a significant difference was found when comparing BvsT2, RMSSD - $p$-value $=0.01$, ln_HF $-p$-value $=$ not significant (n.s.), QSE - $p$-value $<0.01$, ln RSA - $p$-value $<0.05$. The comparison between $\mathrm{CG}$ and $\bar{E} \bar{G}$ did not show any main effect associated with groups when performing repeated measures ANOVA with group as a factor.

The right panel of Figure 1, depicts the group trends for EG which is substantially different with respect to the behavior reported for CG. Vagal tone estimators stay stable for every tilt point, a slight but not significant decrease is shown by RSA in the comparison BvsT1. The absence of any difference is confirmed by the results of repeated measures ANOVA. Results reported in Table I show lack of significance when performing the within-subjects analysis testing each variable of interest separately.

Differences in baseline values between the groups are not significant due to large individual variability and small number of subjects.

\section{DISCUSSIONS AND CONCLUSION}

In this study, different measures of vagal tone in response to head up tilt have been employed to investigate the behavior of subjects with or without prenatal exposure to alcohol and smoking. Results for unexposed infants show, as expected, evidence of a physiological vagal withdrawal in response to head-up tilt. These changes with tilt were significant for all four measures. In contrast, changes in the four measures of vagal tone were not significant in the exposed infant.

The reported results for RMSSD in CG are consistent with findings by Myers et al. [8], which showed a significant decrease in beat-to-beat variability as consequence of tilt. The presented results for HF power are in accordance with previous studies [6] showing a vagal withdrawal in a population of adults in response to tilt.

Complexity analysis performed in this study shows, for unexposed infants, a significant within-subjects decrease of QSE. This trend is similar to previous findings reporting Approximate Entropy (ApEn) decreasing as a consequence of vagal blockade [16]. The decrease in complexity on such short-term scale $(\mathrm{m}=1)$ may indicate a shift towards increased regularity of RR series due to vagal withdrawal and increase prevalence of sympathetic system.

The quantification of vagal tone by means of RSA is still largely debated. Polyvagal theory by Porges [17] states that successful vagal regulation is marked by RSA suppression or withdrawal, which is thought to facilitate an organism's ability to cope with challenging state. On the other hand, high correlation between RSA measures and breathing frequency have been reported, suggesting a substantial equivalence of the two quantities. In this study, absence of correlation between the above-mentioned parameters has been reported for each tilt block. Instead, significant correlation between RSA and HF power is consistent. Given these findings it is not possible to conclude if the RSA could provide a reliable estimation of vagal tone estimation or it may eventually mimic the behavior of HF power modulated by respiratory frequency. At the same time, RSA trend is consistent with the reported estimators of vagal magnitude.

TABLE I. MEAN \pm STD OF PARAMETER FOR EACH TILT BLOCK AND WITHIN-SUBJECTS RESULTS OF REPEATED MEASURES ANOVA FOR CG AND EG. ACROSS-SUBJECTS DIFFERENCES ARE FOUND IN CG ONLY AS A CONSISTENT RESULTS FOR EACH OF THE TESTED PARAMETERS.

\begin{tabular}{|c|c|c|c|c|c|c|c|}
\hline \multicolumn{4}{|c|}{ Control Group (N=15) } & \multicolumn{4}{|c|}{ Exposed Group (N=13) } \\
\hline $\mathrm{B}$ & $\mathrm{T} 1$ & $\mathrm{~T} 2$ & $\mathrm{~T} 3$ & $\mathrm{~B}$ & $\mathrm{~T} 1$ & $\mathrm{~T} 2$ & $\mathrm{~T} 3$ \\
\hline \multicolumn{8}{|c|}{ In $R M S S D$} \\
\hline$-4.27 \pm 0.69$ & $-4.50 \pm 0.62$ & $-4.59 \pm 0.68$ & $-4.58 \pm 0.67$ & $-4.17 \pm 0.53$ & $-4.19 \pm 0.60$ & $-4.18 \pm 0.49$ & $-4.20 \pm 0.57$ \\
\hline \multicolumn{2}{|c|}{$p$-value $=0.001$} & \multicolumn{2}{|c|}{$\mathrm{F}(1.83,25.55)=9.29$} & \multicolumn{2}{|c|}{$p$-value $=$ n.s. } & \multicolumn{2}{|c|}{$\mathrm{F}(2.11,25.30)=0.05$} \\
\hline \multicolumn{8}{|c|}{$\ln H F$} \\
\hline$-10.46 \pm 1.45$ & $-10.76 \pm 1.27$ & $-11.04 \pm 1.48$ & $-10.92 \pm 1.41$ & $-10.26 \pm 1.03$ & $-10.26 \pm 1.16$ & $-10.20 \pm 0.99$ & $-10.19 \pm 1.15$ \\
\hline \multicolumn{2}{|c|}{$p$-value $<0.05$} & \multicolumn{2}{|c|}{$\mathrm{F}(2.40,33.52)=3.22$} & \multicolumn{2}{|c|}{$p$-value $=$ n.s. } & \multicolumn{2}{|c|}{$\mathrm{F}(2.10,25.19)=0.07$} \\
\hline \multicolumn{8}{|c|}{ QSE } \\
\hline $3.72 \pm 0.74$ & $3.57 \pm 0.58$ & $3.44 \pm 0.66$ & $3.46 \pm 0.73$ & $3.89 \pm 0.53$ & $3.80 \pm 0.58$ & $3.74 \pm 0.50$ & $3.78 \pm 0.52$ \\
\hline \multicolumn{2}{|c|}{$p$-value $<0.001$} & \multicolumn{2}{|c|}{$\mathrm{F}(2.58,36.09)=7.66$} & \multicolumn{2}{|c|}{$p$-value $=$ n.s. } & \multicolumn{2}{|c|}{$F(1.86,22.37)=0.71$} \\
\hline \multicolumn{8}{|c|}{$\ln R S A$} \\
\hline$-3.93 \pm 0.77$ & $-4.28 \pm 0.74$ & $-4.35 \pm 0.84$ & $-4.29 \pm 0.77$ & $-3.99 \pm 0.61$ & $-4.06 \pm 0.72$ & $-3.95 \pm 0.61$ & $-3.90 \pm 0.67$ \\
\hline \multicolumn{2}{|c|}{$p$-value $<0.05$} & \multicolumn{2}{|c|}{$\mathrm{F}(1.69,23.66)=4.24$} & $p$-valu & & \multicolumn{2}{|c|}{$\mathrm{F}(1.80,21.53)=1.02$} \\
\hline
\end{tabular}



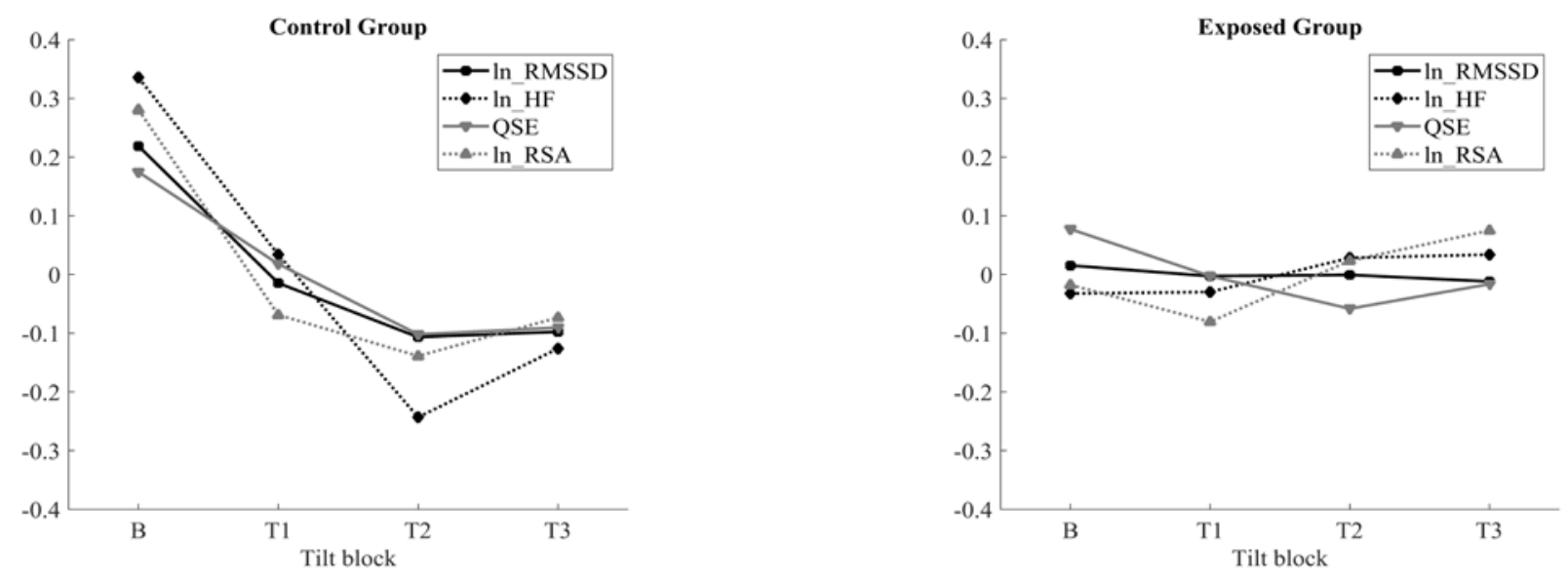

Figure 1. Left and right panel show the mean values of the four extracted parameters and their trends when plotted based upon the tilt blocks. CG show a consistent behavior across all indexes, characterized by a decrease from B to T3. Group means in EG do exhibit a flatter tendency. The mean value for a given index (obtain by grouping means in B, T1, T2, T3) has been subtracted from mean values reported in Table I to provide a comparable scale across parameters.

The results for EG show consistent lower vagal withdrawal in response to tilt challenge for every extracted parameter. In literature, prenatal exposure to smoke has been reported to effectively impair receptors in the medullary 5HT system, as well as alterations in cardiorespiratory control mechanisms and abnormalities in the pathogenesis of the parasympathetic systems [3]. It is possible to speculate that illustrated difference in vagal tone comparing $\mathrm{CG}$ and $\mathrm{EG}$ may effectively arise as a consequence of prenatal exposure and may contribute to the decreased infant's ANS capability to maintain homeostatic control when expose to a direct physiological challenge. The reported findings seems to confirm the altered heart rate response to tilt as described for other groups of infants at high risk for SIDS [18].

The reported absence of group differences in the comparison between CG and EG is possibly related to the limited database analyzed and remarkable variability between subjects.

In future analyses this same approach will be applied to a larger cohort with the aim of investigating the individual effects of alcohol and smoking as well as quantitative estimates of prenatal exposures.

\section{ACKNOWLEDGMENT}

This research was supported by grants U01HD055154, U01HD045935, U01HD055155, U01HD045991 and U01AA016501 issued by the National Institute on Alcohol Abuse and Alcoholism, Eunice Kennedy Shriver National Institute of Child Health and Human Development, and the National Institute on Deafness and Other Communication Disorders.

\section{REFERENCES}

R. F. Carlin and R. Y. Moon, "Risk factors, protective factors, and current recommendations to reduce sudden infant death syndrome a review," JAMA Pediatr., vol. 171, no. 2, pp. 175-180, Feb. 2017.

[2] W. G. Guntheroth and P. S. Spiers, "The Triple Risk Hypotheses in Sudden Infant Death Syndrome," Pediatrics, vol. 110, no. 5, pp. e64-e64, 2002.

[3] J. R. Duncan et al., "Prenatal nicotine-exposure alters fetal autonomic activity and medullary neurotransmitter receptors: implications for sudden infant death syndrome," J. Appl. Physiol., vol. 107, no. 5, pp. 1579-1590, Nov. 2009.

J. J. K. Jaakkola and M. Gissler, "Maternal Smoking in
Pregnancy, Fetal Development, and Childhood Asthma," Am. J. Public Health, vol. 94, no. 1, pp. 136-140, 2004.

[5] R. M. Harper and H. C. Kinney, "Potential Mechanisms of Failure in the Sudden Infant Death Syndrome.," Curr. Pediatr. Rev., vol. 6, no. 1, pp. 39-47, 2010.

[6] N. Montano, T. G. Ruscone, A. Porta, F. Lombardi, M. Pagani, and A. Malliani, "Power spectrum analysis of heart rate variability to assess the changes in sympathovagal balance during graded orthostatic tilt.," Circulation, vol. 90, no. 4, pp. 1826-31, Oct. 1994.

[7] P. Graziano and K. Derefinko, "Cardiac vagal control and children's adaptive functioning: a meta-analysis.," Biol. Psychol., vol. 94, no. 1, pp. 22-37, Sep. 2013.

[8] M. M. Myers et al., "Cardiorespiratory physiology in the safe passage study: protocol, methods and normative values in unexposed infants," Acta Paediatr. Int. J. Paediatr., vol. 106, no. 8, pp. 1260-1272, 2017.

[9] M. Malik, "Heart rate variability," Eur. Heart J., vol. 17, pp. 354 381, 1996.

[10] G. G. Berntson, J. T. Cacioppo, and K. S. Quigley, "Respiratory sinus arrhythmia: Autonomic origins, physiological mechanisms, and psychophysiological implications," Psychophysiology, vol. 30, no. 2, pp. 183-196, Mar. 1993.

[11] K. A. Dukes et al., "The safe passage study: Design, methods, recruitment, and follow-up approach," Paediatr. Perinat. Epidemiol., vol. 28, no. 5, pp. 455-465, 2014.

[12] J. R. Isler, T. Thai, M. M. Myers, and W. P. Fifer, "An automated method for coding sleep states in human infants based on respiratory rate variability.," Dev. Psychobiol., vol. 58, no. 8, pp. 1108-1115, 2016.

[13] D. E. Lake, "Renyi entropy measures of heart rate Gaussianity," IEEE Trans. Biomed. Eng., vol. 53, no. 1, pp. 21-27, 2006.

[14] R. P. Bartsch, A. Y. Schumann, J. W. Kantelhardt, T. Penzel, and P. C. Ivanov, "Phase transitions in physiologic coupling," Proc. Natl. Acad. Sci., vol. 109, no. 26, pp. 10181-10186, 2012.

[15] B. Kralemann, M. Rosenblum, and A. Pikovsky., "DAMOCO : MATLAB toolbox for multivariate data analysis, based on coupled oscillators approach," pp. 1-8, 2011.

[16] J. Penttilä et al., "Effect of cardiac vagal outflow on complexity and fractal correlation properties of heart rate dynamics," Auton. Autacoid Pharmacol., vol. 23, no. 3, pp. 173-179, 2003.

[17] S. W. Porges, J. A. Doussard-Roosevelt, A. L. Portales, and S. I. Greenspan, "Infant regulation of the vagal 'brake' predicts child behavior problems: A psychobiological model of social behavior," Dev. Psychobiol., vol. 29, no. 8, pp. 697-712, Dec. 1996.

[18] A. Edner, M. Katz-Salamon, H. Lagercrantz, and J. Milerad, "Heart rate response profiles during head upright tilt test in infants with apparent life threatening events," Arch Dis Child, vol. 76, pp. 27-30, 1997. 